\title{
Hipotireoidismo e sua associação com transtornos depressivos: uma revisão de
}

\section{literatura}

\author{
Hypothyreoidism and its association with depressive disorders: a literature review \\ Hipotiroidismo y su associacion com transtornos depressivos: revisión de la literatura
}

Recebido: 12/08/2021 | Revisado: 17/08/2021 | Aceito: 18/08/2021 | Publicado: 22/08/2021

\author{
Antônio Ricardo Neto \\ ORCID: https://orcid.org/0000-0001-6647-9374 \\ Centro Universitário de Patos de Minas, Brasil \\ E-mail: antonioneto12@unipam.edu.br \\ Bárbara Queiroz de Figueiredo \\ ORCID: https://orcid.org/0000-0003-1630-4597 \\ Centro Universitário de Patos de Minas, Brasil \\ E-mail: barbarafigueiredo@unipam.edu.br \\ Rúbia Carla Oliveira \\ ORCID: https://orcid.org/0000-0002-7162-3497 \\ Centro Universitário de Patos de Minas, Brasil \\ E-mail: rubiacoliveira@unipam.edu.br
}

\begin{abstract}
Resumo
Introdução: A função tireoidiana está intimamente associada às funções neuropsicológicas, incluindo o estado mental e as funções cognitivas. O hipotireoidismo subclínico (HSC) é definido como uma condição com níveis elevados de hormônio estimulador da tireoide (TSH) e níveis normais de tiroxina livre (T4). Objetivo: evidenciar a relação entre hipotireoidismo e transtorno depressivo. Metodologia: trata-se de uma pesquisa descritiva do tipo revisão integrativa. A pesquisa foi realizada através do acesso online nas bases de dados National Library of Medicine (PubMed MEDLINE), Scientific Electronic Library Online (Scielo), Cochrane Database of Systematic Reviews (CDSR), Google Scholar, EBSCO Information Services, no mês de agosto de 2021. Resultados: Ainda que não seja claro o papel desempenhado pelos hormônios tireoidianos na fisiopatologia dos transtornos mentais, tem sido sugerido que pequenas mudanças nos níveis de hormônio da tireoide, mesmo dentro da faixa normal, podem estar relacionadas à alteração da função cerebral na depressão. Atualmente, existem 2 hipóteses explicativas: o déficit de serotonina e o déficit de noradrenalina no sistema nervoso central provocados pelos distúrbios hormonais. É importante ressaltar que a via tireoidiana-psíquica é bidirecional, portanto, tanto alterações tireoidianas podem provocar sintomas depressivos ou exacerbar uma patologia psiquiátrica prévia, quanto a depressão pode promover distúrbios tireoidianos, sendo este segundo caso menos frequente. Conclusão: O papel da função tireoidiana nas doenças depressivas é pouco claro. Embora existam algumas evidências de que discretas alterações tireoidianas predisponham a casos de depressão, as anormalidades específicas envolvendo a tireoide e os quadros depressivos permanecem pouco conhecidas. No entanto, existe um vínculo de causalidade.
\end{abstract}

Palavras-chave: Hipotireoidismo; Hipófise; Tiroxina; Serotonina.

\begin{abstract}
Introduction: Thyroid function is closely associated with neuropsychological functions, including mental state and cognitive functions. Subclinical hypothyroidism (HSC) is defined as a condition with elevated levels of thyroid stimulating hormone (TSH) and normal levels of free thyroxine (T4). Objective: to demonstrate the relationship between hypothyroidism and depressive disorder. Methodology: this is a descriptive integrative review type research. The research was carried out through online access in the National Library of Medicine databases (PubMed MEDLINE), Scientific Electronic Library Online (Scielo), Cochrane Database of Systematic Reviews (CDSR), Google Scholar, EBSCO Information Services, in August 2021. Results: Although the role played by thyroid hormones in the pathophysiology of mental disorders is unclear, it has been suggested that small changes in thyroid hormone levels, even within the normal range, may be related to altered brain function in depression. Currently, there are 2 explanatory hypotheses: the serotonin deficit and the norepinephrine deficit in the central nervous system caused by hormonal disturbances. It is important to emphasize that the thyroid-psychic pathway is bidirectional, therefore, both thyroid alterations can cause depressive symptoms or exacerbate a previous psychiatric pathology, and depression can promote thyroid disorders, this second case being less frequent. Conclusion: The role of thyroid function in depressive illness is unclear. Although there is some evidence that mild thyroid alterations predispose to cases of depression, the specific abnormalities involving the thyroid and depressive conditions remain poorly known. However, there is a causal link.
\end{abstract}

Keywords: Hypothyroidism; Hypophysis; Thyroxine; Serotonin. 


\begin{abstract}
Resumen
Introducción: La función tiroidea está estrechamente relacionada con las funciones neuropsicológicas, incluido el estado mental y las funciones cognitivas. El hipotiroidismo subclínico (HSC) se define como una condición con niveles elevados de hormona estimulante de la tiroides (TSH) y niveles normales de tiroxina libre (T4). Objetivo: demostrar la relación entre hipotiroidismo y trastorno depresivo. Metodología: se trata de una investigación tipo revisión descriptiva integradora. La investigación se llevó a cabo a través del acceso en línea en las bases de datos de la Biblioteca Nacional de Medicina (PubMed MEDLINE), Scientific Electronic Library Online (Scielo), Cochrane Database of Systematic Reviews (CDSR), Google Scholar, EBSCO Information Services, en agosto de 2021. Resultados: Aunque el papel que juegan las hormonas tiroideas en la fisiopatología de los trastornos mentales no está claro, se ha sugerido que pequeños cambios en los niveles de hormonas tiroideas, incluso dentro del rango normal, pueden estar relacionados con la función cerebral alterada en la depresión. Actualmente, existen 2 hipótesis explicativas: el déficit de serotonina y el déficit de noradrenalina en el sistema nervioso central provocado por alteraciones hormonales. Es importante destacar que la vía tiroidea-psíquica es bidireccional, por tanto, ambas alteraciones tiroideas pueden provocar síntomas depresivos o exacerbar una patología psiquiátrica previa, y la depresión puede promover trastornos tiroideos, siendo este segundo caso menos frecuente. Conclusión: el papel de la función tiroidea en la enfermedad depresiva no está claro. Aunque existe alguna evidencia de que las alteraciones leves de la tiroides predisponen a los casos de depresión, las anomalías específicas que involucran la tiroides y las condiciones depresivas siguen siendo poco conocidas. Sin embargo, existe un vínculo causal.
\end{abstract}

Palabras clave: Hipotiroidismo; Hipófisis; Tiroxina; Serotonina.

\title{
1. Introdução
}

O hormônio tireotrofina (TSH) é uma glicoproteína circulante no sangue e que estimula a produção de outros hormônios pela tireoide. No entanto, a síntese do TSH é regulada através do hormônio estimulador de tireotrofina (TRH) produzido pelo hipotálamo. Os níveis de TSH e TRH, de maneira inversa, são proporcionais ao nível do hormônio tireoidiano. Quando há presença de um alto nível de hormônio tireoidiano no sangue, uma menor quantidade de TRH é liberada pelo hipotálamo e, por consequência, uma menor quantidade do TSH é secretada pela glândula. Assim, a ação contrária ocorrerá quando houver presença de uma menor quantidade de hormônio tireoidiano na corrente sanguínea. Esse processo é reconhecido como um mecanismo de retroalimentação negativa, sendo responsável pela conservação dos níveis apropriados destes hormônios na corrente sanguínea (Barros et al. 2018).

Os distúrbios da tireoide acometem a maioria da população brasileira, de maneira sintomática ou assintomática. A Triidoritonina (T3) e tiroxina (T4) são hormônios sintetizados pela glândula endócrina tireoide e o distúrbio provocada por essas substâncias, acarretam no surgimento do hipertireoidismo e hipotireoidismo, na qual podem causar inúmeras alterações no organismo (Soares et al. 2020). A ação primária dos hormônios tireoidianos é estimular as células ao consumo de oxigênio. No hipotireoidismo, a produção dos hormônios torna-se abaixo do normal, induzindo a metabolismo lento, o que provoca o surgimento de alguns sintomas, como bradicardia e ganho de peso (Fernandes et al. 2018).

O hipotireoidismo primário é um problema clínico comumente encontrado. O tratamento é indicado se o hormônio estimulador da tireoide (TSH) for superior a $10 \mathrm{uIU} / \mathrm{ml}$. A causa mais comum de hipotireoidismo é a tireoidite autoimune. O hipotireoidismo é considerado uma condição inflamatória caracterizada por níveis elevados de citocinas inflamatórias, como proteína C reativa (PCR), interleucina-6 (IL 6) e fator de necrose tumoral alfa (TNF- $\alpha$ ). Marcadores inflamatórios podem ter um papel na patogênese de muitas complicações associadas ao hipotireoidismo, causando disfunção endotelial, proliferação e migração de células musculares lisas e recrutando e ativando células inflamatórias. Além disso, eles induzem a produção de interferon-gama e também mediar a apoptose (Tayde et al. 2017).

Curiosamente, níveis elevados de anticorpos antitireoidianos foram documentados em pacientes com depressão clínica. A prevalência de positividade de anticorpos antitireoidianos em pacientes deprimidos é de 20\%, em oposição a 5\% $10 \%$ na população em geral (Wolkowitz et al. 2003). A descoberta de que a depressão costuma coexistir com a tireoidite subclínica autoimune sugere que a depressão também pode ser causada por alterações no sistema imunológico ou vice-versa ou que, na verdade, pode ser um distúrbio autoimune. Foi constatado que pacientes com depressão têm biomarcadores 
aumentados de inflamação, incluindo citocinas imunes inatas, proteínas de fase aguda, quimiocinas, e moléculas de adesão. É uma possibilidade de que a depressão possa pertencer ao espectro de distúrbios inflamatórios e degenerativos. Da mesma forma, o hipotireoidismo é um estado pró-inflamatório caracterizado por citocinas elevadas e múltiplos sintomas depressivos (Anisman, 2009). Sob essa perspectiva, o objetivo deste estudo é demonstrar a possível relação do hipotireoidismo com a depressão.

\section{Metodologia}

Trata-se de uma pesquisa descritiva do tipo revisão integrativa da literatura, que buscou evidenciar a relação entre hipotireoidismo e transtorno depressivo. A pesquisa foi realizada através do acesso online nas bases de dados National Library of Medicine (PubMed MEDLINE), Scientific Electronic Library Online (Scielo), Cochrane Database of Systematic Reviews (CDSR), Google Scholar, Biblioteca Virtual em Saúde (BVS) e EBSCO Information Services, no mês de agosto de 2021. Para a busca das obras foram utilizadas as palavras-chaves presentes nos descritores em Ciências da Saúde (DeCS): em inglês: "hypothyroidism", "TSH", "T4", "pituitary", "depression", em português: "hipotireoidismo", "TSH", "T4", "hipófise", "depressão" e em espanhol: "hipotiroidismo", "TSH", "T4", "pituitaria", "depresión"

Como critérios de inclusão, foram considerados artigos originais, que abordassem o tema pesquisado e permitissem acesso integral ao conteúdo do estudo, publicados no período de 2003 a 2021, em inglês, português e espanhol. O critério de exclusão foi imposto naqueles trabalhos que não estavam em inglês, português e espanhol que não tinham passado por processo de Peer-View e que não relacionassem a depressão e a hipotireoidismo. A estratégia de seleção dos artigos seguiu as seguintes etapas: busca nas bases de dados selecionadas; leitura dos títulos de todos os artigos encontrados e exclusão daqueles que não abordavam o assunto; leitura crítica dos resumos dos artigos e leitura na íntegra dos artigos selecionados nas etapas anteriores. Assim, totalizaram-se 28 artigos científicos para a revisão integrativa da literatura, com os descritores apresentados acima. Após esta seleção, filtraram-se por artigos dos últimos dois anos e por artigos em línguas portuguesa, inglesa e espanhola.

\section{Resultados e Discussão}

A função tireoidiana está intimamente associada às funções neuropsicológicas, incluindo o estado mental e as funções cognitivas (Feldman et al. 2013). O hipotireoidismo subclínico (HSC) é definido como uma condição com níveis elevados de hormônio estimulador da tireoide (TSH) e níveis normais de tiroxina livre (T4) (Baumgartner et al. 2014). A prevalência de HSC é de aproximadamente 4-10\% em adultos e está associada à disfunção neuropsicológica (Demartini et al. 2014). Nos últimos anos, um número crescente de estudos investigou a associação entre HSC e depressão (Fjaellegaard et al. 2015). No entanto, as conclusões desses relatórios não chegaram a um consenso. Alguns estudos relataram que a prevalência de depressão é maior em pessoas com HSC do que em eutireoidianos (Yu et al. 2016), enquanto outros descobriram que o risco de depressão é comparável entre as duas populações (Kim et al. 2018).

Vários estudos investigaram se a presença de autoimunidade tireoidiana com níveis de hormônio tireoidiano dentro da faixa de referência está associada à depressão. É reconhecido que eventos estressantes podem exacerbar ou desencadear episódios de doenças autoimunes, e também parece haver uma associação entre autoimunidade tireoidiana e depressão pósparto. Dois estudos mostraram uma associação entre autoimunidade tireoidiana e depressão: estudo de Dayan et al. (2012) que mostrou uma associação em 583 mulheres entre os níveis de anticorpos antiperioxidase tireoidiana (TPO)> $100 \mathrm{mU} / 1$ e os escores da Escala de Depressão de Edimburgo, e Kirim et al. 2012 que mostrou uma associação em 201 indivíduos com positividade para anticorpos TPO e pontuações altas na Escala de Avaliação de Depressão de Hamilton. 
Ainda que não seja claro o papel desempenhado pelos hormônios tireoidianos na fisiopatologia dos transtornos mentais, tem sido sugerido que pequenas mudanças nos níveis de hormônio da tireoide, mesmo dentro da faixa normal, podem estar relacionadas à alteração da função cerebral na depressão. Atualmente, existem 2 hipóteses explicativas: o déficit de serotonina e o déficit de noradrenalina no sistema nervoso central provocados pelos distúrbios hormonais. É importante ressaltar que a via tireoidiana-psíquica é bidirecional, portanto, tanto alterações tireoidianas podem provocar sintomas depressivos ou exacerbar uma patologia psiquiátrica prévia, quanto a depressão pode promover distúrbios tireoidianos, sendo este segundo caso menos frequente (Almeida et al. 2013).

A conexão entre disfunção tireoidiana evidente, humor anormal e transtorno cognitivo foi bem documentada. $\mathrm{O}$ hipotireoidismo evidente é uma causa de transtorno de humor importante, incluindo melancolia, e pode resultar em demência em deterioração. Recentemente, vários relatórios enfocaram o hipotireoidismo subclínico (HSC) e seus resultados neuropsiquiátricos e neurocognitivos latentes; no entanto, esses estudos não conseguiram determinar a natureza precisa e a força dessa relação. HSC ou "hipotireoidismo leve" é caracterizado por níveis elevados de hormônio estimulador da tireoide (TSH) com hormônios da tireoide circulantes normais (FT4) e é uma disfunção usual do eixo da tireoide. O HSC pode afetar até 17,6 por cento dos pacientes na população em geral (Shan, 2016). Vários relatórios demonstraram uma conexão entre HSC e sintomas depressivos atuais, depressão maior atual e uma história de depressão maior ao longo da vida. Enquanto isso, outros trabalhos não conseguiram detectar essa associação. Além disso, a relação entre sintomas depressivos e HSC é controversa, principalmente em pacientes mais velhos, pois a prevalência de HSC aumenta com a idade. O HSC pode afetar até $22 \%$ das mulheres> 60 anos e é um pouco menos prevalente em homens (Villar et al. 2007).

A importância dos hormônios da tireoide no desenvolvimento e funcionamento do cérebro é destacada pelas consequências neurológicas devastadoras da deficiência grave de iodo, mutações no gene MCT8 e hipotireoidismo congênito não tratado Friesema et al. 2004). Além disso, percebe-se a importância dos transportadores do hormônio tireoidiano e das desiodinases de iodotironina na entrega dos hormônios para onde atuam (Schwartz et al. 2005). Em estudos com animais, os hormônios tireoidianos influenciam a neurotransmissão noradrenérgica e serotonérgica, que desempenham um papel fundamental na patogênese da depressão e são alvos para as terapias antidepressivas atuais. Estudo de Bauer et al. 2002 mostrou níveis aumentados de serotonina no córtex cerebral de ratos após a administração de T3 e também diminuição da síntese de serotonina no cérebro com hipotireoidismo. A serotonina também tem um efeito inibitório na secreção do hormônio liberador de tireotropina (TRH), sugerindo um ciclo de feedback que permite a ativação do eixo hipotálamo-hipófise-tireoide quando os níveis de serotonina no cérebro estão baixos. Em humanos, o nível plasmático de serotonina se correlaciona positivamente com as concentrações de T3, mostrando-se aumentado no hipertireoidismo. Desse modo, com a redução dos hormônios tireoidianos na vigência do tratamento antitireoidiano, costuma ocorrer a redução nos níveis séricos da serotonina.

Além de uma associação entre a função tireoidiana e a depressão nesta população, também foi demonstrado de forma convincente que os indivíduos em uso de tiroxina apresentam um bem-estar psicológico mais pobre do que seus homólogos sem doença tireoidiana, mesmo quando incluindo apenas indivíduos cuja reposição é considerada bioquimicamente adequada. Esse achado sugere ainda uma associação entre a função tireoidiana e a depressão (Heuer et al. 2007). Vários estudos têm mostrado consistentemente um pior bem-estar psicológico em indivíduos em uso de tiroxina, mesmo quando se considera apenas aqueles indivíduos cuja reposição de tiroxina é considerada bioquimicamente adequada. Um estudo do Kramer et al. 2009 em idosos não encontrou aumento da depressão, mas um uso significativamente maior de antidepressivos naqueles em uso de tiroxina.

A associação entre o uso de tiroxina e o comprometimento do bem-estar psicológico foi replicada em pelo menos 3 ocasiões e parece robusta. Isso contrasta com a falta de associação entre a função tireoidiana e o bem-estar psicológico em grandes estudos comunitários. Uma possibilidade é a atribuição incorreta de sintomas devido a uma causa separada de baixo- 
astral à doença tireoidiana descoberta acidentalmente, seguida por tratamento com hormônio tireoidiano, resultando em bemestar psicológico aparente e mais pobre de indivíduos sob uso de tiroxina. A falta de melhora dos sintomas com tiroxina nesses indivíduos resulta em um "ciclo de atribuição incorreta". Tanto a depressão quanto o hipotireoidismo bioquímico subclínico são comuns, particularmente em uma população feminina mais velha. Portanto, há uma prevalência razoável de sobreposição entre as duas condições (Panicker et al. 2009).

No estudo de Andrade Júnior et al. (2010), foi demonstrada que a presença concomitante de ansiedade e depressão foi cinco vezes maior entre os casos do que entre os controles. A ocorrência de sintomas ansiosos foi cerca de três vezes maior entre os casos (40\%) em relação aos controles (14\%), enquanto a prevalência de sintomas depressivos mostrou-se $75 \%$ superior entre casos (28\%) quando comparada aos controles (16\%). Desse modo, esse estudo caso-controle apontou uma maior probabilidade de pacientes com hipotireoidismo apresentarem sintomas ansiosos e depressivos em comparação a controles eutireoidianas. Já em estudo de Oliveira et al. (2001), com o intuito de confirmar esta assertiva, foram revisados prontuários de 117 pacientes adultos, consultando em ambulatório de Endocrinologia, com diagnóstico de hipotireoidismo primário. A amostra é foi constituída por 9 homens (idade média 53,4 anos) e 108 mulheres (44,9 anos), com hipotireoidismo: póscirúrgico $(n=37)$, espontâneo clássico $(n=45)$ ou subclínico $(n=35)$. Os sintomas psiquiátricos pesquisados foram os utilizados no diagnóstico de transtornos depressivos pelo DSM-IV. Foram caracterizados 3 grupos de pacientes: com diagnóstico de depressão em acompanhamento psiquiátrico com ou sem antidepressivos ( $n=15)$; sem sintomas psiquiátricos $(n=34)$ e com sintomas sugestivos de depressão $(n=68)$. Desses 68 pacientes, 15, 23, 21, 7 e 2 apresentaram, respectivamente, 1, 2, 3, 4 e 5 sintomas psiquiátricos. Dois indivíduos apresentavam 5 sintomas psiquiátricos e 28 apresentavam 3 ou 4 sintomas, números esses respectivamente associados com depressão maior e distimia.

Em concomitância, segundo Bahls et al. (2004), o T4 plasmático, total e livre, tem sido encontrado como normal ou aumentado em pacientes deprimidos. Aproximadamente, $20 \%$ a 30\% têm níveis acima do limite normal. Mesmo dentro da faixa normal, as taxas de $T_{4}$ tendem a diminuir assim que remite a depressão. Na depressão há um aumento de $\mathrm{T} 4$, que pode ser explicado pela hipercortisolemia, haja vista que cortisol estimularia o hipotálamo a produzir o hormônio estimulador de tireotropina (TRH) e, com isso, haveria um estímulo da função tireoidiana. A hipercortisolemia deve-se a um defeito no funcionamento do hipocampo, o que leva a um efeito inibidor do eixo hipotálamohipófise. Por isso, em alguns casos de depressão, uma desconexão funcional entre o hipotálamo e outras áreas do cérebro removeria a influência inibitória do hipocampo que iria favorecer a hipercortisolemia levando a um aumento do T4 (Abreu, 2011).

A associação entre transtornos de humor e alterações no eixo hipotálamo-hipófise-tireoide (HHT) foi reconhecida, embora isso seja frequentemente relatado em indivíduos com manifestações do hipotireoidismo (Constant et al. 2006). No sistema nervoso central, alterações nos níveis de hormônios, como somatostatina e serotonina, podem resultar em distúrbios neuropsiquiátricos (Ortiga et al. 2016). As evidências existentes também sugerem que esses mecanismos podem afetar potencialmente o eixo HHT e, portanto, explicar a associação entre hipotireoidismo e depressão. Vários estudos relataram uma redução no nível de somatostatina no líquido cefalorraquidiano, levando ao aumento do nível de TSH entre indivíduos com depressão (Najafi et al. 2015). Por outro lado, a deficiência de serotonina, que também é comumente vista em pessoas com depressão, foi postulada como causadora de alterações no eixo HHT.

Tradicionalmente, a depressão é relatada entre pessoas de 35 a 45 anos. É importante ressaltar que se tornou cada vez mais comum em idosos, pois a própria senescência está associada a alterações bioquímicas no eixo HHT. A secreção de hormônios tireoidianos é reduzida com o aumento da idade, com um nível de T3 mais baixo, mas uma concentração do hormônio T4 relativamente inalterada (Chakera et al. 2012). Em comparação com a população mais jovem, o nível de TSH mais alto é visto em idosos devido à degradação reduzida do T4 e sua conversão periférica para o hormônio T3, com subsequente feedback positivo para o eixo HHT (Bauer et al. 2008). Indivíduos com hipotireoidismo manifesto experimentam 
uma grande variedade de sinais e sintomas clínicos, incluindo intolerância ao frio, ganho de peso, disfunção cognitiva e distúrbios do humor. É importante notar que apenas até 30\% dos indivíduos com hipotireoidismo compartilham características clínicas semelhantes (Joffe et al. 2013), com o grupo de idosos apresentando queixas ainda menos e mais sutis, o que resulta em um diagnóstico tardio de depressão (Davis et al. 2003).

\section{Conclusão}

O papel da função tireoidiana nas doenças depressivas é pouco claro. Embora existam algumas evidências de que discretas alterações tireoidianas predisponham a casos de depressão, as anormalidades específicas envolvendo a tireoide e os quadros depressivos permanecem pouco conhecidas. Após anos de pesquisas, permanece pouco esclarecida a importância da relação entre o eixo HHT e as depressões, assim como os mecanismos subjacentes às alterações tireoidianas encontradas nos pacientes deprimidos. Portanto, mais pesquisas serão necessárias para uma melhor compreensão do papel do eixo HHT na patogênese e no tratamento dos quadros depressivos (Bahls et al. 2004).

Embora a maioria dos deprimidos tenha níveis circulantes de T3, T4 e TSH normais, existem evidências de atividade alterada do eixo HHT em alguns casos de depressão, que incluem; 1) aumento de T4 total e/ou livre, muitas vezes, dentro dos limites da normalidade. É característico o achado de T4 e/ou T4L plasmático elevado sem alterações de T3; 2) resposta exagerada do TSH ao desafio com TRH em $10 \%$ e resposta diminuída em $25 \%$ dos pacientes; 3 ) níveis elevados de anticorpos antitireoidianos presentes em 15\% dos casos; e 4) concentração elevada de TRH no líquido céfalo-raquidiano de deprimidos. Sob essa perspectiva, nota-se que o hipotireoidismo pode estar relacionado aos transtornos depressivos, sendo de suma importância que futuras pesquisas atestem tal causalidade.

\section{Referências}

Abreu, G. P. P. (2011). A importância da tiroide nas perturbações da mente. [Dissertação para obtenção do Grau de Mestre em Medicina]. Covilhão: Universidade da Beira Interior.

Almeida, M. M. R., Kuwae, A. S., Quirino, C. M. J., Gondim, L. V. \& Silva, D. O. F. (2013). A depressão e sua relação com o hipotireoidismo. Revista de Medicina e Saúde de Brasília, 2 (3), 164-169.

Andrade Júnior, N. E., Pires, M. L. E. \& Thuler, L. C. S. (2010). Sintomas depressivos e ansiosos em mulheres com hipotireoidismo. Revista Brasileira de Ginecologia e Obstetrícia, 32 (7), 321-227.https://doi.org/10.1590/S0100-72032010000700003

Anisman, H. (2009). Efeitos em cascata de estressores e ativação do sistema imunológico inflamatório: Implicações para transtorno depressivo maior. $J$ Psychiatry Neurosci., 34 (1), 4-20.

Bahls, S. C. \& Carvalho, G. A. (2004). A relação entre a função tireoidiana e a depressão: uma revisão. Brazilian Journal of Psychiatry, 26 (1), 4048. https://doi.org/10.1590/S1516-44462004000100012

Barros, A. C. S., Xavier, E. M., Reis, I. S., Carvalho, P. R. B., Oliveira, R. S., Pacheco, F.K. \& Queissada, D. D. (2018). Farmacêutico bioquímico: uma abordagem voltada para o TSH e doenças da tireoide. Revista Saúde e Desenvolvimento Humano, 6 (1), 67-74.

Bauer, M., Goetz, T., Glenn, T., Whybrow, P. C. (2008). A interação tireóide-cérebro em distúrbios da tireóide e distúrbios do humor. J Neuroendocrinol., 20 (10): 1101-1114

Bauer, M., Heinz, A. \& Whybrow, P. C. (2002). Hormônios tireoidianos, serotonina e humor: de sinergia e significado no cérebro adulto. Mol Psychiatry, 7 (1), 140-156.

Baumgartner, C., Blum, M. R. \& Rodondi, N. (2014). Hipotireoidismo subclínico: resumo das evidências em 2014. Swiss Med Wkly, 144 (1458), 1-32

Chakera, A. J., Pearce, S. H. \& Vaidya, B. (2012). Tratamento para hipotireoidismo primário: abordagens atuais e possibilidades futuras. Drug Des Devel Ther.,6 (1), 1-11

Constant, E. L., Adam, S., Seron, X., Bruyer, R., Seghers, A. \& Daumerie, C. (2006). Hipotireoidismo e depressão maior: uma disfunção executiva comum? J Clin Exp Neuropsychol., 28 (5): 790-807

Davis, J. D., Stern, R. A. \& Flashman, L. A. (2003). Aspectos cognitivos e neuropsiquiátricos do hipotireoidismo subclínico: significado em idosos. Curr Psychiatry Rep., 5 (5): 384-390.

Dayan, C. M. \& Pânico, V. (2013). Hipotireoidismo e depressão. European Thyroid Journal, 2 (1), 168-179. https://doi.org/10.1159/000353777 
Demartini, B., Ranieri, R., Masu, A., Selle, V., Scarone, S. \& Gambini, O. (2014). Sintomas depressivos e transtorno depressivo maior em pacientes afetados por hipotireoidismo subclínico um estudo transversal. J Nerv Mental Dis., 202 (1), 603-607.

Feldman, A. Z., Shrestha, R. T. \& Hennessey, J. V. (2013). Manifestações neuropsiquiátricas de doenças da tireoide. Endocrinol Metab Clin North Am., 42 (1), 453-76.

Fernandes, G. Q. \& Freitas, G. G. (2018). Prevalência de hipotireoidismo em pacientes com diabetes mellitus tipo 2. Revista de Medicina, 97(3), 273-277.

Fjaellegaard, K., Kvetny, J., Allerup, P. N., Bech, P. \& Ellervik, C. (2015). Bem-estar e depressão em indivíduos com hipotireoidismo subclínico e autoimunidade tireoidiana - um estudo populacional geral. Nord J Psychiatry, 69 (1), 73-78.

Friesema, E. C., Grueters, A., Biebermann, H., Krude, H., von Moers, A., Reeser, M., Barrett, T. G., Mancilla, E. E., Svensson, J., Kester, M. H., Kuiper, G. G., Balkassmi, S., Uitterlinden, A. G., Koehrle, J., Rodien, P., Halestrap, A. P. \& Visser, T. J. (2004). Associação entre mutações em um transportador de hormônio da tireoide e retardo psicomotor ligado ao X severo. The Lancet, 364 (1), 1435-1437.

Heuer, H. (2007). A importância dos transportadores do hormônio tireoidiano para o desenvolvimento e funcionamento do cérebro. Best Pract Res Clin Endocrinol Metab, 21 (1), 265-276.

Joffe, R. T., Pearce, E. N., Hennessey, J. V., Ryan, J. J. \& Stern. R. A. (2013). Hipotireoidismo subclínico, humor e cognição em idosos: uma revisão. Int J Geriatr Psychiatry, 28 (2): 111-118

Kim, J. S., Zhang, Y., Chang, Y., Ryu, S., Guallar, E. \& Shin, Y. C. (2018). Hipotireoidismo subclínico e depressão incidente em adultos jovens e de meiaidade. J Clin Endocrinol Metab, 103 (1), 1827-1833

Kirim, S., Keskek, S. O., Koksal, F., Haydardedeoglu, F. E., Bozkirli, E. \& Toledano, Y. (2012). Depressão em pacientes com tireoidite autoimune crônica eutireoidiana. Endocrinology Journal, 59 (1), 705-708.

Kramer, C. K., von Muhlen, D., Kritz-Silverstein, D. \& Barrett-Connor, E. (2009). Hipotireoidismo tratado, função cognitiva e humor deprimido na velhice: o estudo Rancho Bernardo. Eur J Endocrinol, 161 (1), 917-921.

Najafi, L., Malek, M., Hadian, A., Ebrahim Valojerdi, A., Khamseh, M. E. \& Aghili, R. (2015). Sintomas depressivos em pacientes com hipotireoidismo subclínico - o efeito do tratamento com levotiroxina: um ensaio clínico duplo-cego randomizado. Endocr Res., 40 (3): $121-6$.

Oliveira, M. C., Filho, A. A. P., Schuch, T. \& Mendonça W. L. (2001). Sinais e sintomas sugestivos de depressão em adultos com hipotireoidismo primário. Arquivos Brasileiros de Endocrinologia e Metodologia, 45 (6), 570-576. https://doi.org/10.1590/S0004-27302001000600011

Ortiga-Carvalho, T. M., Chiamolera, M. I., Pazos-Moura, C. C. \& Wondisford, F. E. (2016). Eixo hipotálamo-hipófise-tireoide. Compr Physiol., 6 (3): 13871428

Panicker, V., Saravanan, P., Vaidya, B., Evans, J., Hattersley, A. T., Frayling, T. M. \& Dayan, C. M. (2009). Variação comum no gene DIO2 prediz bem-estar psicológico inicial e resposta à terapia combinada de tiroxina mais triiodotironina em pacientes com hipotireoidismo. J Clin Endocrinol Metab, 94 (1), 1623 1629 .

Schwartz, C. E., May, M. M., Carpenter, N. J., Rogers, R. C., Martin, J., Bialer, M. G., Ward, J., Sanabria, J., Marsa, S., Lewis, J. A., Echeverri, R., Lubs, H. A., Voeller, K., Simensen, R. J. \& Stevenson, R. E. (2005). Allan-Herndon-Dudley síndrome e o gene transportador de monocarboxilato 8 (MCT8). Am J Hum Genet, 77 (1), 41-53.

Shan, Z. (2016). Status do iodo e prevalência de distúrbios da tireoide após a introdução da iodização universal obrigatória do sal por 16 anos na China: um estudo transversal em 10 cidades. Thyroid, 26 (1), 1125-1130

Soares, G. V. D., Soares, C. V. D., Medeiros, T. K. F. \& Santos, E. B. (2020). Physiological disorders related to the thyroid gland: a literary review. Research, Society and Development, 9 (7), 1-11.

Tayde, P. S., Bhagwat, N. M., Sharma, P., Sharma, B., Dalwadi, P. P., Sonawane, A., Subramanyam, A., Chadha, M. \& Vartthakavi, P. K. (2017). Hipotireoidismo e depressão: as citocinas são o elo? Indian Journal of Endocrinology, 21 (6), 886-892.

Villar, H. C., Saconato, H., Valente, O. \& Atallah, A. N. (2007). Thyroid hormone replacement for subclinical hipotireoidism. Cochrane Database Syst. Rev., 2007 (3), 1-21.

Wolkowitz, O. M. \& Rothschild, A. J. (2003). Psychoneuroendocrinology: The Scientific Basis of Clinical Practice. $1^{\text {a }}$ ed. Washington, DC, EUA: American Psychiatric.

Yu, J., Tian, A. J., Yuan, X. \& Cheng, X. X. (2016). Hipotireoidismo subclínico após tratamento com 131I da doença de Graves: um fator de risco para depressão? PLoS ONE, 11 (46), 1-9 